CARDIOVASCULAR MEDICINE

\title{
Insulin induced increase in coronary flow reserve is abolished by dexamethasone in young men with uncomplicated type 1 diabetes
}

\author{
H Laine, J Sundell, P Nuutila, O T Raitakari, M Luotolahti, T Rönnemaa, T Elomaa, P Koskinen, \\ J Knuuti
}

See end of article for authors' affiliations

.....................

Correspondence to: Dr J Sundell, Turku PET Centre, Turku University Central Hospital, PO Box 52, FIN-20521 Turku, Finland; jan.sundell@utu.fi

Accepted

2 September 2003

\begin{abstract}
Objective: To examine the role of the sympathetic nervous system in regulating insulin's action on coronary perfusion in uncomplicated type 1 diabetes by blocking centrally mediated sympathetic activity with dexamethasone.

Methods: Positron emission tomography and oxygen 15 labelled water were used to quantify myocardial blood flow basally and during adenosine infusion with or without simultaneous euglycaemic physiological hyperinsulinaemia in nine non-smoking men with type 1 diabetes and 12 healthy non-diabetic men. Each patient was studied both with and without previous dexamethasone treatment for two days $(2 \mathrm{mg} /$ day). Results: Insulin increased coronary flow reserve in diabetic (from $4.3(0.7)$ to $5.1(0.6), \mathrm{p}<0.05$ ) and non-diabetic (from $4.3(0.3)$ to $5.4(0.4), \mathrm{p}<0.05)$ patients. In contrast to non-diabetic patients dexamethasone pretreatment abolished the insulin induced increase in coronary flow reserve in diabetic patients $(p<0.05)$ leading to lower coronary flow reserve in diabetic than in non-diabetic patients (3.9 (0.6) v $7.1(0.9), p<0.05)$.

Conclusions: These results show that insulin's ability to modulate coronary perfusion is sustained in young patients with type 1 diabetes without microvascular complications or autonomic neuropathy. Dexamethasone treatment abolished the insulin induced increase in coronary flow reserve in diabetic patients but not in healthy study participants, suggesting that sympathetic activation plays an important part in regulating insulin's effects on myocardial perfusion in patients with type 1 diabetes.
\end{abstract}

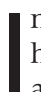
nsulin induces a dose and time dependent vasodilatation in healthy people..$^{1-3}$ We have recently shown that insulin can also enhance myocardial blood flow in patients with type 1 diabetes. ${ }^{4}$ Type 1 diabetes is an important risk factor for the development of coronary artery disease, and cardiovascular disease is the leading cause of death in diabetic patients. ${ }^{5}$ Glucose-insulin-potassium infusion (GIK) has been found to be beneficial in the treatment of acute myocardial ischaemia, especially in diabetic patients. ${ }^{6}$ It has been shown with single photon emission tomography that GIK improves regional myocardial perfusion and function mainly in segments adjacent to the recently infarcted area. ${ }^{7}$ Thus, in addition to insulin actions on substrate metabolisms, ${ }^{8}$ insulin induced coronary vasodilatation may partly contribute to the known beneficial effect of GIK on myocardial ischaemia.

Insulin induces vasodilatation through the sympathetic nervous system ${ }^{9}$ and the endothelium dependent mechanism including the L-arginine-nitric oxide pathway. ${ }^{10}$ The mechanisms of insulin induced vasodilatation are well characterised but have been studied mainly in the peripheral vasculature. Since differences in the regulation of vasodilatation between coronary and peripheral arteries have been observed, ${ }^{11}$ previous studies targeting insulin's effects on the skeletal muscle vasculature cannot be applied directly to the coronary vasculature. Therefore, insulin's cardiovascular actions have been widely studied in recent years.

In addition to the standard risk factors, autonomic neuropathy ${ }^{12}$ has been suggested to contribute to the increased occurrence of cardiovascular disease in diabetic patients. At physiological concentrations insulin modulates cardiac autonomic control by producing cardiac vagal withdrawal and inducing a relative hypersympathetic tone. ${ }^{14}{ }^{15}$
Cardiac sympathetic signals have been suggested to play an important part in regulating myocardial perfusion. ${ }^{16}$ Recent evidence from positron emission tomography (PET) studies indicates that cardiac sympathetic pathways may be altered in a substantial number of diabetic patients with the absence of apparent autonomic neuropathy as determined by autonomic reflex tests. ${ }^{17-19}$ These findings raise the possibility that the role of the sympathetic nervous system in regulating myocardial perfusion and insulin's effects on it may be altered in diabetic patients.

PET enables quantitative and accurate measurements of myocardial blood flow non-invasively in humans. Recently, coronary flow reserve measured with PET was compared with flow reserve measured with transthoracic Doppler echocardiography, with good agreement between the methods. ${ }^{20}$ In the present PET study we examined whether regulation of insulin induced coronary vasodilatation is altered in patients with uncomplicated type 1 diabetes. Dexamethasone treatment has been previously shown to abolish insulin induced sympathetic activation. ${ }^{21}$ Therefore, the role of the sympathetic nervous system in regulating insulin's action on coronary perfusion was tested by blocking centrally mediated sympathetic activation with dexamethasone for two days. Myocardial blood flow, hyperaemic adenosine stimulated flow, and coronary flow reserve were determined after an overnight fast basally and during euglycaemic physiological

\footnotetext{
Abbreviations: GIK, glucose-insulin-potassium infusion; [ $\left[{ }^{15} \mathrm{O}\right] \mathrm{CO}$, oxygen- 15 labelled carbon monoxide; $\left[{ }^{15} \mathrm{O}^{\mathrm{H}} \mathrm{H}_{2} \mathrm{O}\right.$, oxygen- 15 labelled water; PET, positron emission tomography
} 
Table 1 Characteristics of the study participants

\begin{tabular}{|c|c|c|}
\hline & Type 1 diabetic & Non-diabetic \\
\hline Number & 9 & 12 \\
\hline Age (years) & $36(3)$ & $34(1)$ \\
\hline Body mass index $\left(\mathrm{kg} / \mathrm{m}^{2}\right)$ & $24.8(0.4)$ & $25.8(0.4)$ \\
\hline $\mathrm{V}_{2} \mathrm{max}(\mathrm{ml} / \mathrm{kg} / \mathrm{min})$ & $32(1)$ & $33(1)$ \\
\hline Blood pressure $(\mathrm{mm} \mathrm{Hg})$ & $120 / 66(3 / 3)$ & $118 / 66(3 / 3)$ \\
\hline Blood $\mathrm{HbA}_{1 \mathrm{lc}}(\%)$ & $8.3(0.6)^{*}$ & $5.3(0.1)$ \\
\hline Total cholesterol $(\mathrm{mmol} / \mathrm{l})$ & $4.7(0.3)$ & $4.8(0.2)$ \\
\hline HDL cholesterol (mmol/l) & $1.52(0.10)$ & $1.41(0.10)$ \\
\hline Triglycerides $(\mathrm{mmol} / \mathrm{l})$ & $0.76(0.08)$ & $0.80(0.11)$ \\
\hline LDL cholesterol (mmol/l/) & $2.84(0.21)$ & $2.89(0.23)$ \\
\hline Apolipoprotein A 1 (g/l) & $1.47(0.07)$ & $1.33(0.05)$ \\
\hline Apolipoprotein B (g/l) & $0.80(0.05)$ & $0.83(0.05)$ \\
\hline Apolipoprotein(a) (U/I) & $128(64)$ & $91(24)$ \\
\hline \multicolumn{3}{|c|}{$\begin{array}{l}\text { Data are mean (SEM). } \\
{ }^{*} \mathrm{p}<0.001 v \text { non-diabetic. } \\
\mathrm{HbA}_{1 c} \text { glycosylated haemoglobin } \mathrm{A}_{1 c i} \mathrm{HDL} \text {, high density lipoprotein; LDL, low density lipoprotein; } \dot{V}_{\mathrm{O}_{2}} \text { max, } \\
\text { maximum aerobic capacity. }\end{array}$} \\
\hline
\end{tabular}

hyperinsulinaemia by using PET and oxygen-15 labelled water $\left(\left[{ }^{15} \mathrm{O}\right] \mathrm{H}_{2} \mathrm{O}\right)$.

\section{METHODS}

\section{Study participants}

Table 1 shows the characteristics of the study participants. Nine non-smoking men with type 1 diabetes were investigated. The mean (SEM) duration of diabetes was 9.6 (1.2) years. None of the diabetic patients had signs or symptoms of any disease other than type 1 diabetes. Retinal photographs, autonomic nerve function tests, and overnight urinary albumin excretion rate measurements were normal in all of the studied patients with type 1 diabetes. The results of the diabetic patients were compared with results of 12 nonsmoking, non-diabetic otherwise matched healthy men. Echocardiographically determined left ventricular mass, dimensions, and function, as well as the stress echocardiograms and ECGs, were normal in all studied patients.

\section{Study design}

Each participants was studied on two separate days, once after the administration of dexamethasone for two days ( $0.5 \mathrm{mg}$, four times a day) with the last dose of dexamethasone on the morning of the PET study, and once without dexamethasone. The two PET study days were in a random order. During dexamethasone treatment the intermediate acting insulin dose was moderately increased and the patients were advised to increase the short acting insulin doses if required. The normal dose of intermediate acting insulin was reduced by a third and short acting insulin was withdrawn from diabetic patients on the PET study morning.

All PET studies were performed after an overnight fast. Additionally, the patients were instructed to avoid all drinks and foods containing caffeine for 12 hours before the PET studies. On each study day myocardial perfusion was measured three times (fig 1), first at rest and then after administration of adenosine ( $140 \mu \mathrm{g} / \mathrm{kg} / \mathrm{min}$ for five minutes intravenously). Thereafter, physiological hyperinsulinaemia $(1 \mathrm{mU} / \mathrm{kg} / \mathrm{min})^{22}$ was started and continued for 60 minutes before the second intravenous infusion of adenosine and the third perfusion measurement. The insulin infusion was continued until the third perfusion measurement was taken. ECG and heart rate were monitored continuously during the studies. Blood pressure was monitored with an automatic oscillometric blood pressure monitor (OMRON HEM-705C, Omron Healthcare, Hamburg, Germany) during the PET study. Each patient gave written informed consent. The study was conducted according to the guidelines of the Declaration
Perfusion measurement

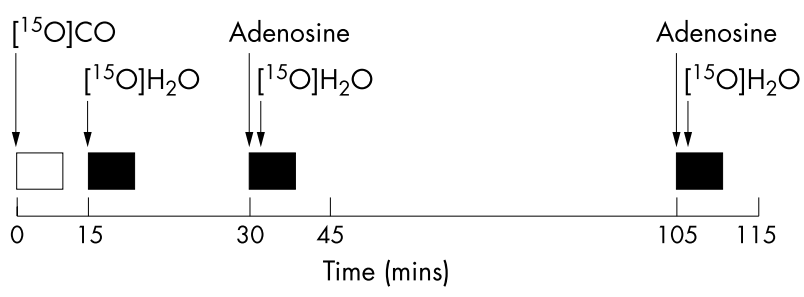

\begin{tabular}{|c|}
\hline $\begin{array}{c}\text { Insulin infusion } \\
(1 \mathrm{mU} / \mathrm{kg} / \mathrm{min})\end{array}$ \\
\hline $20 \%$ glucose infusion \\
\hline
\end{tabular}

Figure 1 Design of the study. Myocardial perfusion was measured with oxygen-15 labelled water $\left(\left[{ }^{[5} \mathrm{O}\right] \mathrm{H}_{2} \mathrm{O}\right)$ three times: once during basal conditions and twice during adenosine stimulation without and with simultaneous insulin infusion. Insulin was infused at a rate of $1 \mathrm{mU} / \mathrm{kg} /$ $\min$.

of Helsinki and the study protocol was accepted by the ethics committee of the Turku University Central Hospital.

\section{Insulin infusion}

Insulin and glucose were infused in a catheter inserted into the right antecubital vein. The left hand was kept in a heated pillow and arterialised venous blood was withdrawn from a heated left antecubital vein. Insulin (Actrapid Human; Novo Nordisk, Copenhagen, Denmark) was infused at rate of $1 \mathrm{mU} / \mathrm{kg} / \mathrm{min}$. Normoglycaemia was maintained by using a variable rate of $20 \%$ glucose. The rate of the glucose infusion was adjusted according to plasma glucose concentrations determined every five minutes from arterialised venous blood. Samples for serum insulin and free fatty acid determination were taken every 30 minutes. Whole body glucose uptake was calculated from the glucose infusion rate after correcting for changes in the glucose pool size. ${ }^{22}$

\section{Production of oxygen 15 labelled carbon monoxide and $\left[{ }^{15} \mathrm{O}\right] \mathrm{H}_{2} \mathrm{O}$}

For production of ${ }^{15} \mathrm{O}$ a low energy deuteron accelerator, Cyclone 3, was used (Ion Beam Application Inc, Louvain-laNeuve, Belgium). Oxygen-15 labelled carbon monoxide $\left(\left[{ }^{15} \mathrm{O}\right] \mathrm{CO}\right)$ was produced in a conventional way. ${ }^{23}\left[{ }^{15} \mathrm{O}\right] \mathrm{H}_{2} \mathrm{O}$ was produced by dialysis techniques in a continuously 
working water module. ${ }^{24}$ Sterility and pyrogenity tests for water and chromatographic analysis for gases were performed to verify the purity of the products.

Image acquisition, processing, and correction

The patients were positioned supine in a 15 slice ECAT 931/ 08-12 tomograph (Siemens/CTI Inc, Knoxville, Tennessee, USA). After the transmission scan, the patients' nostrils were closed and they inhaled $\left[{ }^{15} \mathrm{O}\right] \mathrm{CO}$ (about $2.5 \mathrm{GBq}$ ) for two minutes through a three way inhalation flap valve $(0.14 \%$ CO mixed with room air). After the inhalation, two minutes was allowed for $\mathrm{CO}$ to combine with haemoglobin in red blood cells before a static scan for four minutes was started. During the scan period, three blood samples were drawn at two minute intervals and blood radioactivity was measured immediately with a well counter (Bicron 3MW3/3, USA). A 10 minute period was allowed for $\left[{ }^{15} \mathrm{O}\right] \mathrm{CO}$ radioactive decay before the flow measurements. Flow was measured at rest and starting 60 seconds after intravenous administration of adenosine. $\left[{ }^{15} \mathrm{O}\right] \mathrm{H}_{2} \mathrm{O}$ (about $1.5 \mathrm{GBq}$ ) was injected intravenously and dynamic scanning was started for six minutes $(6 \times 5$ s, $6 \times 15$ s, $8 \times 30 \mathrm{~s})$. All data were corrected for dead time, decay, and photon attenuation and reconstructed into a $128 \times 128$ matrix. The final in-plane resolution in reconstructed and Hann filtered (0.3 cycles/s) images was $9.5 \mathrm{~mm}$ (full width half maximum).

\section{Calculation of regional myocardial blood flow and coronary flow reserve}

Regions of interest were drawn in the lateral, anterior, and septal wall of the left ventricle in four representative transaxial slices in each study as previously described. ${ }^{25}$ The regions of interest outlined in the baseline images were copied to the images obtained after adenosine administration. Values of regional myocardial blood flow (expressed as $\mathrm{ml} / \mathrm{g}$ of tissue/minute) were calculated according to the previously published method by the single compartment model. ${ }^{26}$ The arterial input function was obtained from the left ventricular time activity curve by a previously validated method, ${ }^{27}$ in which corrections were made for the limited recovery of the left ventricular region of interest and the spillover from the myocardial signals. No regional differences were found in myocardial perfusion. Therefore, to enhance accuracy and statistical analysis of the flow measurements, the average blood flow of global myocardium was calculated and used in further analysis.

The basal coronary flow reserve was defined as a ratio of the myocardial blood flow during adenosine infusion to the flow at rest. The insulin stimulated flow reserve was defined as the ratio of the myocardial blood flow during simultaneous adenosine and insulin infusions to the flow at rest.

\section{Retinal photography}

Retinas were photographed after mydriatic instillation with a Canon CR4-45NM fundus camera (Canon, Kanagawa, Japan). One $45 \%$ field photograph, including areas of papilla and macula, was taken from each eye. Polaroid photo prints were analysed by one experienced diabetologist (TR).

\section{Echocardiographic examination}

To rule out silent ischaemia and cardiomyopathy, study participants underwent a rest and a bicycle exercise echocardiographic examination. All echocardiographic recordings and analyses were performed by the same experienced investigator (ML) with a commercially available ultrasound scanner (Acuson 128XP/10, Acuson Inc, Mountain View, California, USA). Standard echocardiographic views of the left ventricle were obtained and cardiac dimensions were measured first at rest. Thereafter, an upright bicycle ergometer exercise test was performed with work load increased by $20 \mathrm{~W}$ at one minute intervals. The test was a symptom limited maximal exercise test and continued until extreme fatigue when at least $90 \%$ of the predicted maximum heart rate was reached. The echocardiograms were recorded before and immediately after the exercise. All patients had a normal exercise capacity, were asymptomatic, and had no diagnostic ST changes in ECGs and no wall motion disturbances either at rest or immediately after the maximal exercise.

\section{Autonomic nerve function tests}

To exclude autonomic neuropathy, diabetic patients underwent a series of standardised non-invasive cardiovascular reflex tests. ${ }^{28}$ Autonomic nerve function tests measuring mainly the parasympathetic control included a deep breathing test, the Valsalva manoeuvre, and an orthostatic test. Diastolic blood pressure response to isometric hand grip was used as the measure of sympathetic autonomic nervous system control. The QT intervals corrected for heart rate were calculated from rest ECG by Bazett's formula.

\section{Analytical methods}

Venous blood samples were taken after 12 hours of overnight fasting. During insulin infusion plasma glucose was determined by the glucose oxidase method. ${ }^{29}$ Serum insulin was measured by radioimmunoassay kit (Pharmacia, Uppsala, Sweden). Serum total cholesterol, high density lipoprotein cholesterol, and triglyceride concentrations were measured by standard enzymatic methods (Boehringer Mannheim GmbH, Mannheim, Germany) with a fully automated analyser (Hitachi 704; Hitachi Ltd, Tokyo, Japan). The low density lipoprotein cholesterol concentration was calculated by the Friedewald formula. ${ }^{30}$ Apolipoprotein A 1 and apolipoprotein B were measured by an immunonephelometric method (Behring BNA, Marburg, Germany). Serum apolipoprotein(a) was determined by a solid phase two site immunoradiometric assay (Mercodia Apo(a) RIA; Mercodia $\mathrm{AB}$, Uppsala, Sweden). Urinary albumin excretion was measured immunonephelometrically (Behring) with antiserum from Dakopatts (Glostrup, Denmark). Microalbuminuria was defined as an albumin excretion rate of $\geqslant 20 \mu \mathrm{g} / \mathrm{min}$ in at least two of the three collected urine samples. Plasma adrenaline (epinephrine) and noradrenaline (norepinephrine) were measured as previously described. ${ }^{31}$

\section{Statistical methods}

The results are expressed as mean (SEM). The effect of dexamethasone treatment on flow rates between the two study days, the responses to adenosine infusion with and without hyperinsulinaemia, and the interaction of these variables were tested by repeated measures analysis of variance (procedure Mixed in Statistical Analysis System, SAS Institute Inc, Cary, North Carolina, USA). Paired and unpaired $t$ tests were used when appropriate. Probability values of $\mathrm{p}<0.05$ were interpreted as significant. All statistical tests were performed with the SAS statistical analysis system (SAS Institute Inc).

\section{RESULTS}

\section{Metabolic and hormonal characteristics}

Serum insulin and free fatty acid concentrations were comparable in the studied groups both in the fasting state and during hyperinsulinaemia on the two study days (table 2). Fasting and hyperinsulinaemic plasma glucose concentrations were higher in diabetic than in non-diabetic patients on both study days. In contrast to non-diabetic participants, in diabetic patients dexamethasone increased both fasting and hyperinsulinaemic glucose concentrations 


\begin{tabular}{|c|c|c|c|c|}
\hline & \multicolumn{2}{|c|}{ Type 1 diabetic } & \multicolumn{2}{|c|}{ Non-diabetic } \\
\hline & Fasting & During clamp & Fasting & During clamp \\
\hline \multicolumn{5}{|c|}{ Plasma glucose (mmol/l) } \\
\hline$d x-$ & $8.3(1.0)^{*}$ & $6.9(0.6)^{*}$ & $5.5(0.1)$ & $5.0(0.1)$ \\
\hline$d x+$ & $12.4(1.1)^{*} \dagger$ & $10.8(1.2)^{\star} \dagger$ & $5.6(0.1)$ & $5.6(0.1)$ \\
\hline \multicolumn{5}{|c|}{ Serum insulin $(\mathrm{mU} / \mathrm{l})$} \\
\hline$d x-$ & $19(3)$ & 71 (3)‡ & $14(1)$ & $59(5) \ddagger$ \\
\hline$d x+$ & $25(6)$ & $62(3) \pm$ & $20(2)$ & $64(6) \ddagger$ \\
\hline \multicolumn{5}{|c|}{ Serum free fatty acids $(\mathrm{mmol} / \mathrm{l})$} \\
\hline$d x-$ & $420(70)$ & $100(30) \ddagger$ & $450(50)$ & $70(20) \ddagger$ \\
\hline$d x+$ & $570(110)$ & $130(30) \ddagger$ & $490(60)$ & $90(10) \dagger \ddagger$ \\
\hline
\end{tabular}

(table 2). Insulin stimulated whole body glucose uptake values were lower in diabetic than in non-diabetic patients before dexamethasone treatment (22.7 (3.9) $v \quad 31.9$ (3.3) $\mu \mathrm{mol} / \mathrm{kg} / \mathrm{min}, \mathrm{p}<0.05)$. Dexamethasone reduced insulin stimulated whole body glucose uptake by $31 \%$ in diabetic patients (to $15.6(2.9) \mu \mathrm{mol} / \mathrm{kg} / \mathrm{min}, \mathrm{p}<0.05$ ) and by $50 \%$ in non-diabetics (to $15.9(2.8) \mu \mathrm{mol} / \mathrm{kg} / \mathrm{min}, \mathrm{p}<0.001$ ). After dexamethasone treatment insulin stimulated whole body glucose uptake was comparable between the groups. Fasting serum cortisol concentration was 303 (23) nmol/l in diabetic patients and 332 (31) nmol/l in non-diabetics (not significant). After dexamethasone treatment serum cortisol concentrations were $<20 \mathrm{nmol} / \mathrm{l}$ in all of the studied patients (without versus with dexamethasone, $\mathrm{p}<0.001$; not significant between the groups).

Before dexamethasone treatment fasting plasma adrenaline concentrations were significantly higher in diabetic than in non-diabetic patients, but no difference was found in adrenaline concentrations during hyperinsulinaemia or after dexamethasone treatment (table 3). Fasting plasma noradrenaline concentrations were comparable in the studied groups on the two study days. Insulin infusion increased plasma noradrenaline and adrenaline concentrations in both groups $(\mathrm{p}<0.05)$. After dexamethasone treatment fasting adrenaline concentrations were significantly reduced in diabetic patients $(p<0.05)$ and the insulin induced increase in noradrenaline concentrations was abolished in nondiabetic patients $(\mathrm{p}<0.05)$ (table 3$)$.

\section{Haemodynamic measurements during PET}

No difference was detected between diabetic and nondiabetic patients in any of the haemodynamic parameters either at rest or during the PET studies on either of the study days (table 4).

Adenosine administration induced a significant increase in heart rate and rate-pressure product both basally and during hyperinsulinaemia in the studied groups. Dexamethasone treatment did not change any of the haemodynamic parameters (table 4).

\section{Myocardial blood flow and coronary flow reserve}

Before dexamethasone treatment basal myocardial blood flow was similar in all studied patients (table 5). A comparable flow increase was obtained in both groups by adenosine infusion at the baseline $(p<0.001)$ (table 5). During adenosine stimulation the myocardial blood flows were similar in the studied groups (not significant). Simultaneous insulin and adenosine infusions further increased myocardial blood flow comparably in both groups ( $\mathrm{p}<0.05$ versus adenosine stimulated, not significant between the groups) (table 5). Concordantly, no significant difference was detected between diabetic and non-diabetic patients in the basal (4.33 (0.70) $v 4.25$ (0.30), not significant) or in the insulin stimulated coronary flow reserves $(5.10(0.63) \vee 5.43(0.42), \mathrm{p}<0.05$ versus basal, not significant between the groups) (fig 2 ).

Dexamethasone treatment did not significantly change either basal or adenosine stimulated myocardial blood flow in the studied groups (without versus with dexamethasone, not significant) (table 5). However, dexamethasone abolished the insulin induced increase in myocardial blood flow in patients with type 1 diabetes $(p<0.05)$ and thus myocardial blood flow during adenosine and insulin infusion was 35\% lower in diabetic than in non-diabetic patients $(p<0.05)$ (table 5$)$. Corresponding findings in coronary flow reserves were observed after dexamethasone treatment (fig 2), since no significant difference was detected in the basal coronary flow reserves $(3.65(0.30) v 5.09(0.71)$, diabetic versus nondiabetic, not significant), whereas dexamethasone abolished the insulin induced increase in coronary flow reserve in diabetic patients $(5.10(0.63) \vee 3.90(0.59)$, without versus with dexamethasone, $\mathrm{p}<0.05)$ but not in non-diabetics (5.43 $(0.42) \vee 7.13(0.86)$, respectively, not significant). Thus, after dexamethasone treatment the insulin stimulated

Table 3 Plasma catecholamine concentrations during positron emission tomographic (PET) studies in diabetic and non-diabetic study patients

\begin{tabular}{|c|c|c|c|c|}
\hline & \multicolumn{2}{|c|}{ Type 1 diabetic } & \multicolumn{2}{|l|}{ Non-diabetic } \\
\hline & Fasting & During clamp & Fasting & During clamp \\
\hline \multicolumn{5}{|c|}{ Plasma adrenaline (nmol/l) } \\
\hline$d x-$ & $0.24(0.03)^{*}$ & $0.39(0.07) \dagger$ & $0.15(0.03)$ & $0.27(0.02) \dagger$ \\
\hline$d x+$ & $0.14(0.03) \ddagger$ & $0.24(0.05) \dagger$ & $0.12(0.02)$ & $0.23(0.03) \dagger$ \\
\hline \multicolumn{5}{|c|}{ Plasma noradrenaline (nmol/li) } \\
\hline$d x-$ & $1.42(0.15)$ & $2.00(0.29) t$ & $1.41(0.17)$ & $2.01(0.17) \dagger$ \\
\hline$d x+$ & $1.17(0.21)$ & $1.97(0.26)^{*} \dagger$ & $1.03(0.11) \neq$ & $1.23(0.09) \ddagger$ \\
\hline
\end{tabular}


Table 4 Haemodynamic data during PET study in diabetic and non-diabetic study patients

\begin{tabular}{|c|c|c|c|c|c|c|}
\hline & \multicolumn{3}{|c|}{ Type 1 diabetic } & \multicolumn{3}{|c|}{ Non-diabetic } \\
\hline & Rest & Adenosine & Adenosine + insulin & Rest & Adenosine & Adenosine + insulin \\
\hline \multicolumn{7}{|c|}{$\begin{array}{l}\text { Heart rate } \\
\text { (beats } / \mathrm{min} \text { ) }\end{array}$} \\
\hline$d x-$ & $62(4)$ & $98(6)^{* *}$ & $100(6)^{* *}$ & $60(3)$ & $97(2)^{* *}$ & $100(3)^{* *}$ \\
\hline$d x+$ & $59(2)$ & $97(4)^{\star *}$ & $102(4)^{* *}$ & $56(2)$ & $95(2)^{* *}$ & $99(3)^{* *}$ \\
\hline \multicolumn{7}{|c|}{$\begin{array}{l}\text { Systolic blood } \\
\text { pressure }(\mathrm{mm} \mathrm{Hg})\end{array}$} \\
\hline$d x-$ & $120(3)$ & $116(3)$ & $122(5)$ & $118(3)$ & $121(5)$ & $124(4)^{*}$ \\
\hline$d x+$ & $119(4)$ & $116(4)$ & $119(4)$ & $121(4)$ & $121(4)$ & $121(4)$ \\
\hline \multicolumn{7}{|c|}{$\begin{array}{l}\text { Diastolic blood } \\
\text { pressure }(\mathrm{mm} \mathrm{Hg})\end{array}$} \\
\hline$d x-$ & $66(3)$ & $65(1)$ & $66(3)$ & $66(3)$ & $65(3)$ & $68(3)$ \\
\hline$d x+$ & $65(2)$ & $63(2)$ & $66(2)$ & $68(3)$ & $64(2)$ & $65(3)$ \\
\hline \multicolumn{7}{|c|}{$\begin{array}{l}\text { Rate pressure product } \\
\text { (systolic blood pressure } \times \text { heart rate) } \\
(\mathrm{mm} \mathrm{Hg} / \mathrm{min})\end{array}$} \\
\hline$d x-$ & $7503(514)$ & $11734(981)^{\star *}$ & $13326(889)^{* *}$ & 7067 (405) & $11464(318)^{* *}$ & $12254(381)^{* *}$ \\
\hline$d x+$ & 7023 (325) & $11236(770)^{* *}$ & $12128(779)^{* *}$ & 6787 (383) & $11480(398)^{* *}$ & $12056(523)^{* *}$ \\
\hline
\end{tabular}

coronary flow reserve was $45 \%$ lower in diabetic patients than in non-diabetics $(\mathrm{p}<0.05)$ (fig 2 ).

\section{DISCUSSION}

In the present study we showed that insulin's ability to enhance coronary flow reserve is sustained in patients with type 1 diabetes. In contrast to non-diabetic participants, dexamethasone treatment abolished the insulin induced increase in coronary flow reserve in patients with type 1 diabetes, suggesting that sympathetic activation has an important role in modulating insulin's action on coronary vasodilatation in diabetic patients.

In previous human studies coronary vasoreactivity has been found to be either normal ${ }^{19}$ or more frequently decreased $^{4173233}$ in diabetic patients. However, most of the previous studies have included both patients with type 1 and patients with type 2 diabetes ${ }^{17}$ or other potentially confounding factors such as diabetic complications, smoking, hypertension, obesity, and lipid abnormalities, ${ }^{17} 3233$ which are known to reduce coronary vasoreactivity further. ${ }^{19}{ }^{34}$ Patients with type 1 diabetes with autonomic neuropathy have been found to have decreased coronary flow reserve, while patients with non-neuropathic type 1 diabetes had unaltered myocardial blood flow and coronary flow reserve. ${ }^{19}$ These findings are concordant with the present study, as we detected no difference in myocardial blood flow or coronary flow reserve between the diabetic patients without complications and non-diabetic participants before dexamethasone treatment.

In the present study insulin's vasodilatory effects were measured during simultaneous adenosine infusion. A significant part of adenosine induced vasodilatation is endothelium dependent. ${ }^{35}$ In contrast to the resting condition, where flow and myocardial work (oxygen consumption) are tightly coupled, during adenosine stimulation metabolic control of myocardial blood flow is lost but endothelial and neurogenic controls are still functional. We showed, consistent with previous studies, ${ }^{4}$ that insulin also induces coronary vasodilatation in patients with type 1 diabetes. Insulin's vasodilatory effects on myocardial blood flow may partly contribute to the beneficial effect of GIK in the treatment of diabetic patients with acute myocardial infarction.

In coronary arteries sympathetic activation induces $\alpha$ adrenoceptor mediated vasoconstriction, which is overridden by vasodilatation induced through $\beta$ receptors. ${ }^{11}$ Insulin's sympathoexcitatory effects are supposed to be mediated at least in part by a central neural action. ${ }^{36}$ Dexamethasone administration offers an experimental model to study the effect of centrally mediated sympathetic activity on vascular function..$^{21}$ Dexamethasone treatment for 48 hours was used in the present study to examine the role of the sympathetic nervous system in insulin's effects on myocardial vasculature. Consistent with the previous studies, in the present study dexamethasone decreased sympathetic activity based on plasma catecholamine concentrations leading to the blunted insulin induced coronary flow reserve in patients with type 1 diabetes. Thus, sympathetic activation has an important role in modulating insulin's action on coronary vasodilatation in diabetic patients. The underlying mechanism for this cannot be directly discerned by the present study. In theory, the sympathetic nervous system may provide a compensatory mechanism for impaired myocardial blood supply in these patients. In the present study sympathetic activity was estimated by plasma catecholamine concentrations, which reflect mainly the systemic sympathetic activity. However, to measure specifically cardiac sympathetic activity

Table 5 Myocardial blood flow $(\mathrm{ml} / \mathrm{g} / \mathrm{min})$ in diabetic and non-diabetic study patients

\begin{tabular}{|c|c|c|c|}
\hline & Basal & Adenosine & Adenosine + insulin \\
\hline \multicolumn{4}{|l|}{ Type 1 diabetic } \\
\hline$d x-$ & $0.80(0.06)$ & $3.21(0.40)^{*}$ & $3.92(0.42)^{*} \dagger$ \\
\hline$d x+$ & $0.84(0.05)$ & $2.92(0.44)^{*}$ & $3.20(0.51)^{\star} \mp$ \\
\hline \multicolumn{4}{|l|}{ Non-diabetic } \\
\hline$d x-$ & $0.75(0.06)$ & $3.12(0.23)^{*}$ & $3.91(0.23)^{*} \dagger$ \\
\hline$d x+$ & $0.73(0.07)$ & $3.46(0.40)^{*}$ & $4.89(0.48)^{*} \dagger$ \\
\hline
\end{tabular}




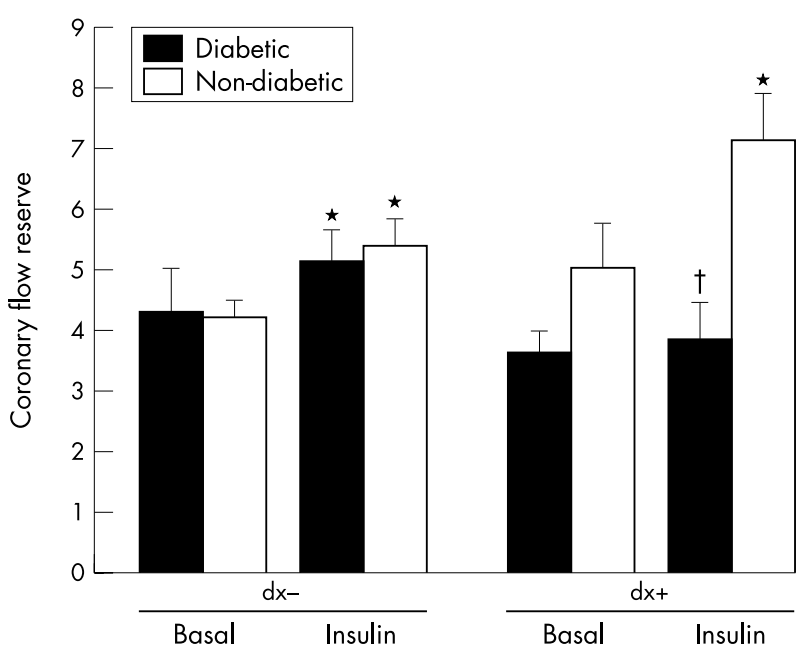

Figure 2 Coronary flow reserve basally and during insulin infusion without $(\mathrm{dx}-)$ and with dexamethasone treatment $(\mathrm{dx}+)$ in patients with and without type 1 diabetes. ${ }^{*} p<0.05 v$ basal, $+p<0.05 v$ nondiabetic.

invasive procedures would have been required, which are unethical in the patients of the present study.

In the present study cardiac sympathetic pathways appeared to be unaltered in patients with type 1 diabetes based on normal autonomic reflex tests. It has been shown recently in patients with type 1 diabetes that the sympathetic stimulation induced increase in coronary flow correlates with cardiac efferent adrenergic signals. ${ }^{17}$ Since dexamethasone pretreatment abolished the insulin induced increase in coronary flow reserve in patients with type 1 diabetes, dexamethasone might have induced a situation mimicking autonomic dysfunction in these patients. The present data also show that the coronary vasculature of young patients with type 1 diabetes is very sensitive to acute changes in the cardiac sympathovagal balance.

In the present study after dexamethasone treatment the diabetic patients had higher plasma glucose concentrations both in the fasting state and during insulin infusion. However, coronary flow reserve did not correlate with plasma glucose concentrations in the fasting state $(r=-0.09)$ or during clamp $(r=0.09)$ in diabetic patients. In addition, we have shown recently by using PET that hyperglycaemia for two days does not alter the coronary flow reserve or insulin induced vasodilatation in patients with uncomplicated type 1 diabetes. ${ }^{4}$ In human studies dexamethasone treatment has also been found to preserve blood vessel responsiveness to different vasodilator stimuli ${ }^{2137}$ also in coronary arteries. ${ }^{3}$ Thus, the reduced coronary flow reserve in patients with type 1 diabetes in the present study after dexamethasone treatment is not explained by the direct effects of dexamethasone on endothelial function since, consistent with the previous studies, in healthy patients the coronary flow reserve was not decreased by dexamethasone.

In summary, the present data show that insulin's ability to increase coronary flow reserve is sustained in young patients with uncomplicated type 1 diabetes. Dexamethasone treatment abolished the insulin induced increase in coronary flow reserve, suggesting that sympathetic activation has an important role in modulating insulin's action on coronary vasodilatation in patients with type 1 diabetes.

\section{ACKNOWLEDGEMENTS}

We thank the staff of the Turku PET Centre for their excellent technical assistance. This study was financially supported by grants of EVO from the Turku University Hospital, the Finnish Foundation for Cardiovascular Research, the Novo Nordisk Foundation, the Research Foundation of Orion Corporation, and the Academy of Finland.

\section{Authors' affiliations}

H Laine*, J Sundell*, P Nuutila*, O T Raitakari, T Elomaa, J Knuuti,

Turku PET Centre, Turku University Central Hospital, Turku, Finland

T Rönnemaa, Department of Medicine, Turku University, Turku, Finland

M Luotolahti, Department of Clinical Physiology, Turku University

P Koskinen, Department of Clinical Chemistry, Turku University

*Also Department of Medicine, Turku University

\section{REFERENCES}

1 Yki-Jarvinen H, Utriainen T. Insulin-induced vasodilatation: physiology or pharmacology? Diabetologia 1998;41:369-79.

2 Sundell J, Nuutila P, Laine $\mathrm{H}$, et al. Dose-dependent vasodilating effects of insulin on adenosine-stimulated myocardial blood flow. Diabetes 2002;51:1125-30.

3 Laine $H$, Nuutila $P$, Luotolahti $M$, et al. Insulin-induced increment of coronary flow reserve is not abolished by dexamethasone in healthy young men. $J$ Clin Endocrinol Metab 2000;85:1868-73.

4 Sundell J, Laine $H$, Nuutila $P$, et al. The effects of insulin and short-term hyperglycemia on myocardial blood flow in young men with uncomplicated Type I diabetes. Diabetologia 2002;45:775-82.

5 Grundy SM, Benjamin IJ, Burke GL, et al. Diabetes and cardiovascular disease: a statement for healthcare professionals from the American Heart Association. Circulation 1999;100:1134-46.

6 Malmberg K, Norhammar A, Wedel H, et al. Glycometabolic state at admission: important risk marker of mortality in conventionally treated patients with diabetes mellitus and acute myocardial infarction: long-term results from the diabetes and insulin-glucose infusion in acute myocardial infarction (DIGAMI) study. Circulation 1999;99:2626-32.

7 Marano L, Bestetti A, Lomuscio A, et al. Effects of infusion of glucose-insulinpotassium on myocardial function after a recent myocardial infarction. Acta Cardiol 2000;55:9-15.

8 Maki M, Luotolahti $M$, Nuutila $P$, et al. Glucose uptake in the chronically dysfunctional but viable myocardium. Circulation 1996;93:1658-66.

9 Scherrer U. Insulin and the regulation of cardiovascular system: role of the Larginine nitric oxide pathway and the sympathetic nervous system. In: Lüscher TE, ed. The endothelium in cardiovascular disease. Heidelberg: Springer Verlag, 1995:108-28.

10 Sobrevia L, Nadal A, Yudilevich DL, et al. Activation of L-arginine transport (system $\mathrm{y}+$ ) and nitric oxide synthase by elevated glucose and insulin in human endothelial cells. J Physiol 1996;490:775-81.

11 Opie LH. The heart, 3rd ed. Philadelphia: Lippincott-Raven, 1998.

12 Ewing DJ, Campbell IW, Clarke BF. Mortality in diabetic autonomic neuropathy. Lancet 1976;i:601-3.

13 Ewing DJ, Boland O, Neilson JM, et al. Autonomic neuropathy, QT interval lengthening, and unexpected deaths in male diabetic patients. Diabetologia $1991 ; 34: 182-5$.

14 Bellavere F, Cacciatori V, Moghetti P, et al. Acute effect of insulin on autonomic regulation of the cardiovascular system: a study by heart rate spectral analysis. Diabet Med 1996;13:709-14.

15 van de $B P$, Hausberg M, Hoffman RP, et al. Hyperinsulinemia produces cardiac vagal withdrawal and nonuniform sympathetic activation in normal subjects. Am J Physiol 1999;276:R178-83.

16 Di Carli MF, Tobes MC, Mangner T, et al. Effects of cardiac sympathetic innervation on coronary blood flow. N Engl J Med 1997;336:1208-15.

17 Di Carli MF, Bianco-Batlles D, Landa ME, et al. Effects of autonomic neuropathy on coronary blood flow in patients with diabetes mellitus. Circulation 1999; 100:813-9.

18 Allman KC, Stevens MJ, Wieland DM, et al. Noninvasive assessment of cardiac diabetic neuropathy by carbon-11 hydroxyephedrine and positron emission tomography. J Am Coll Cardiol 1993;22:1425-32.

19 Stevens MJ, Raffel DM, Allman KC, et al. Cardiac sympathetic dysinnervation in diabetes: implications for enhanced cardiovascular risk. Circulation 1998;98:961-8.

20 Saraste $M$, Koskenvuo J, Knuuti J, et al. Coronary flow reserve: measurement with transthoracic Doppler echocardiography is reproducible and comparable with positron emission tomography. Clin Physiol 2001;21:114-22.

21 Scherrer U, Vollenweider P, Randin D, et al. Suppression of insulin-induced sympathetic activation and vasodilation by dexamethasone in humans. Circulation 1993;88:388-94.

22 Defronzo RA, Tobin JD, Andres R. Glucose clamp technique: a method for quantifying insulin secretion and resistance. Am J Physiol 1979;237:E214-23.

23 Clark JC, Crouzel C, Meyer GJ, et al. Current methodology for oxygen-15 production for clinical use. Int J Rad Appl Instrum [A] 1987;38:597-600.

24 Crouzel C, Clark J, Brihaye C, et al. Radiochemistry automation for PET. In: Stöckling G, Pike V, eds. Radiopharmaceuticals for positron emission tomography. Dordrecht: Kluwer Academic, 1993:45-90. 
25 Laine H, Raitakari OT, Niinikoski H, et al. Early impairment of coronary flow reserve in young men with borderline hypertension. J Am Coll Cardiol 1998;32:147-53.

26 lida H, Takahashi A, Tamura Y, et al. Myocardial blood flow: comparison of oxygen-15-water bolus injection, slow infusion and oxygen-15-carbon dioxide slow inhalation. J Nucl Med 1995;36:78-85.

27 lida $H$, Rhodes CG, de Silva $R$, et al. Use of the left ventricular time-activity curve as a noninvasive input function in dynamic oxygen-15-water positron emission tomography. J Nucl Med 1992;33:1669-77.

28 Piha SJ. Cardiovascular autonomic reflex tests: normal responses and agerelated reference values. Clin Physiol 1991;11:277-90.

29 Kadish A, Little R, Sternberg J. A new and rapid method for the determination of glucose by measurement of rate of oxygen consumption. Clin Chem 1968;14:116-31.

30 Friedewald WT, Levy RI, Fredrickson DS. Estimation of the concentration of low-density lipoprotein cholesterol in plasma, without use of the preparative ultracentrifuge. Clin Chem 1972;18:499-502.

31 Scheinin M, Karhuvaara S, Ojala-Karlsson P, et al. Plasma 3,4 dihydroxyphenylglycol (DHPG) and 3-methoxy-4- hydroxyphenylglycol (MHPG) are insensitive indicators of alpha 2-adrenoceptor mediated regulation of norepinephrine release in healthy human volunteers. Life Sci $1991 ; 49: 75-84$.

32 Nitenberg A, Valensi P, Sachs R, et al. Impairment of coronary vascular reserve and $A C h$-induced coronary vasodilation in diabetic patients with angiographically normal coronary arteries and normal left ventricular systolic function. Diabetes 1993;42:1017-25

33 Pitkanen OP, Nuutila P, Raitakari OT, et al. Coronary flow reserve is reduced in young men with IDDM. Diabetes 1998;47:248-54.

34 Akasaka T, Yoshida K, Hozumi T, et al. Retinopathy identifies marked restriction of coronary flow reserve in patients with diabetes mellitus. J Am Coll Cardiol 1997;30:935-41.

35 Buus NH, Bottcher M, Hermansen F, et al. Influence of nitric oxide synthase and adrenergic inhibition on adenosine-induced myocardial hyperemia. Circulation 2001;104:2305-10.

36 Scherrer U, Sartori C. Insulin as a vascular and sympathoexcitatory hormone: implications for blood pressure regulation, insulin sensitivity, and cardiovascular morbidity. Circulation 1997;96:4104-13.

37 Takeshita A, Mark AL. Decreased vasodilator capacity of forearm resistance vessels in borderline hypertension. Hypertension 1980;2:610-6.

\section{IMAGES IN CARDIOLOGY}

\section{Pericardial constriction after a stab wound to the chest}

A 34 year old taxi driver was admitted to hospital after having been repeatedly stabbed in the chest with a screwdriver by a client. On arrival at the accident department he was in sinus rhythm, and his systolic blood pressure was $95 \mathrm{~mm} \mathrm{Hg}$. The stab wound to the anterior chest divided a costal cartilage. He was resuscitated with intravenous fluids, treated with antibiotics, analgesia, and tetanus toxoid injection. Chest radiography did not show cardiomegaly. Echocardiography was not performed. He was discharged home well after 12 days.

Sixteen years later he was readmitted having had numerous admissions with "heart failure" in the previous 10 years. He described a long history of leg swelling, decreased exercise tolerance, and orthopnoea. On examination he was in atrial fibrillation, with a heart rate of 90 beats/ min; his venous pressure was elevated to $10 \mathrm{~cm}$ above the sternal angle and rose on inspiration. Auscultation of the precordium revealed a pericardial knock; blood pressure was 100/70 mm Hg with no paradox. Chest examination revealed signs of a right pleural effusion. There was pronounced

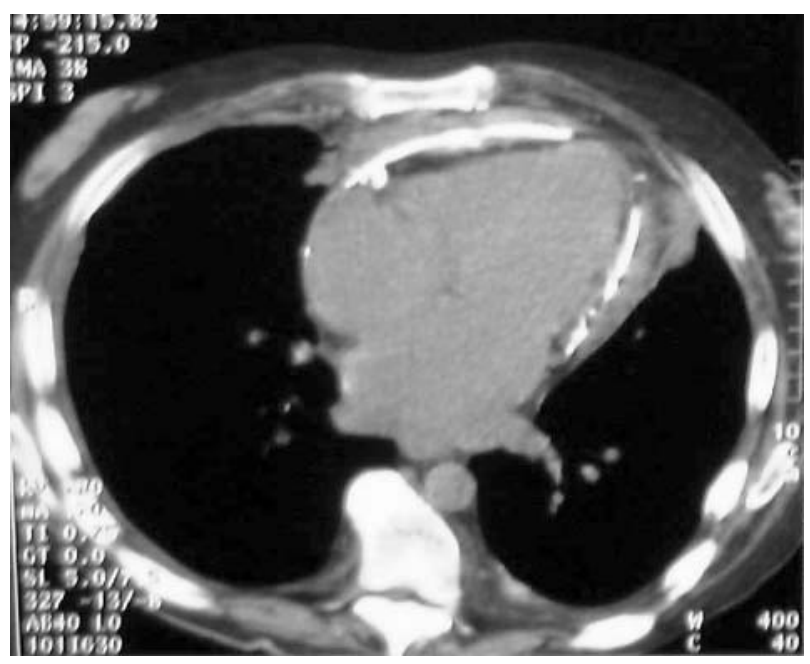

peripheral, sacral, and scrotal oedema. A diagnosis of constrictive pericarditis was made on the clinical findings. Computed tomographic scanning showed dense pericardial calcification (below left) and haemodynamic data supported this diagnosis, demonstrating equivalence of left and right ventricular diastolic pressures (below right).

Pericardectomy was performed; at surgery the pericardium was found to be $1 \mathrm{~cm}$ thick and heavily calcified in places, forming a definite constriction over the right atrium and right ventricle. Two layers of calcium were found with a layer of altered blood in between suggesting the aetiology of the constriction to be haemopericardium. Haemopericardium is rare after stabbing, being reported as infrequently as $0.3 \%$ of cases in one series; constrictive pericarditis is a rare complication of haemopericardium.

A Swallow R D Thomas rosieswallow@hotmail.com

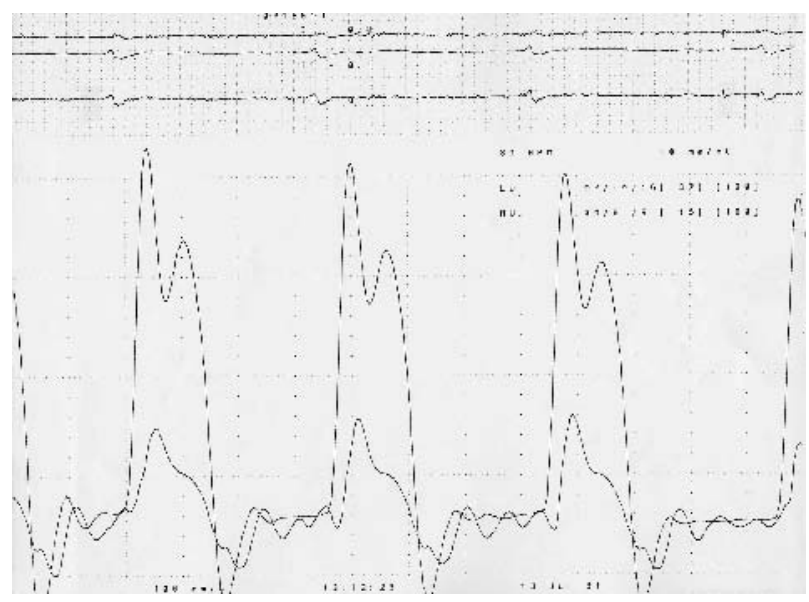

